\title{
Identifying social media user demographics and topic diversity with computational social science: a case study of a major international policy forum
}

\author{
John Brandt ${ }^{1}$ (D) Kathleen Buckingham ${ }^{1} \cdot$ Cody Buntain $^{2} \cdot$ Will Anderson $^{1}$. \\ Sabin Ray ${ }^{1} \cdot$ John-Rob Pool $^{1} \cdot$ Natasha Ferrari $^{1}$
}

Received: 19 June 2019 / Accepted: 26 December 2019 / Published online: 7 January 2020

(c) The Author(s) 2020, corrected publication 2020

\begin{abstract}
When the world's countries agreed on the 2030 Agenda for Sustainable Development, they recognized that equity and inclusion should be at the center of implementing the 17 Sustainable Development Goals (SDGs). SDG 15, which calls for protecting, restoring, and promoting the sustainable use of terrestrial ecosystems, has spurred commitments to restore 350 million hectares of land by 2030. These commitments, primarily made in a top-down manner at the international scale, must be implemented by actively engaging individual landholders and local communities. Ensuring that diverse and marginalized audiences are engaged in the land restoration movement is critical to equitably distributing the economic benefits of restoration. This publication uses social network analysis and machine learning to understand how important the voices of Africans, women, and young people are in governing restoration in Africa. We analyze location- and machine learning-identified demographics from Twitter data collected during the Global Landscapes Forum (GLF), which is the world's largest platform for promoting sustainable land use practices. Our results suggest that convening the GLF in Nairobi, Kenya elevated the voices of African leaders in comparison to the previous GLF in Bonn, Germany. We also found significant demographic differences in topic-level engagement between different ages, races, and genders. The primary contributions of this paper are a novel methodology for quantifying demographic differences in social media engagement and the application of social media and social network analysis to provide critical insights into the inclusivity of a large political conference aimed at engaging youth and African voices.
\end{abstract}

Keywords Text mining $\cdot$ Social media analysis $\cdot$ Demographic analysis $\cdot$ Network

John Brandt

john.brandt@wri.org

1 World Resources Institute, 10 G St NE \#800, Washington DC 20002, USA

2 University of Maryland, College Park, College Park, MD 20742, USA 


\section{Introduction}

Twenty-eight African countries have committed to begin restoring 113 million hectares of degraded land by 2030. These commitments, made to the global Bonn Challenge and the Africa-led African Forest Landscape Restoration Initiative (AFR100), have captured global interest. Events like the Global Landscapes Forum (GLF) bring together policymakers, implementers, and others working to manage land sustainably. In August 2018, the GLF hosted its first Africa-based conference in Nairobi, Kenya to "[bring] together actors from all backgrounds and sectors [and] spark a global conversation around Africa's landscapes" [1]. This regional conference, "Landscape Restoration in Africa: Prospects and Opportunities," aimed to highlight African leadership in the global restoration movement, which historically has largely been led by Western non-governmental organizations and the United Nations. The need to promote the voices of diverse audiences in sustainable development planning and implementation has recently received attention [2,3]. However, quantitatively measuring the success of these initiatives is difficult. Relying on mechanical sensors that detect face-to-face interactions, previous work has demonstrated significant degrees of homophily at academic conferences, suggesting that these types of events may not be the best way to engage diverse audiences [4]. By focusing attention on social media as a proxy, this paper establishes a quantitative method using online discussions to measure the engagement of targeted demographic groups during an event of major environmental importance.

\section{Africa's restoration movement}

Across the continent of Africa, about 300 million people living in drylands depend on the land for income and sustenance. By 2030, this number is expected to increase to 540 million. At the same time, climate change could result in the expansion of Africa's drylands by as much as $20 \%$ [5], as land temperature rises more quickly than the global average [6]. Landscape restoration, the process of returning vitality to a degraded landscape, provides a unique opportunity to mitigate the effects of land degradation, while also improving the economy and human well-being. In recent years, restoration has gained prominence in the international environmental agenda. The United Nations General Assembly launched the UN Decade on Ecosystem Restoration from 2021 to 2030, aiming to create new opportunities for job creation, food security and climate change adaptation on previously degraded land. Global platforms such as the United Nations' Sustainable Development Goals, the Aichi Biodiversity Targets [7], as well as goalbased targets, like the New York Declaration on Forests and the Bonn Challenge have continued to put restoration at the center stage of international and multilateral agreements [8]. 


\section{Communication channels in Africa}

African communication networks have grown with the population and with development. With over 200 million people between the ages of 15 and 24, Africa has the youngest population in the world [9]. Young Africans have grown up in the digital era and are creating the fastest-growing mobile phone market in the world. Over $40 \%$ of Africans own a mobile phone, and 725 million are projected to have a smartphone by 2020 [10]. While mobile phones serve as the primary platform for Internet access, only $37.3 \%$ percent of the population is online [11].

Kenya, which hosted the GLF's African event, has one of the Africa's most developed telecommunications and media networks, but its citizens act like most African Internet users by going online through their phones. Eighty-six percent of Kenya's 50 million residents use the Internet, of which $79 \%$ access the Internet through mobile devices [10]. Kenyan president Uhuru Kenyatta maintains active Facebook and Twitter accounts, and his supporters say his modern communication tactics are "demystifying the presidency" [12]. In Kenya, voters under the age of 35 made up $51 \%$ of the entire electorate in the 2017, and the number of voters in the 26-35 age group has more than doubled since 2013. The rate of digital growth in Africa is staggering. In 2017, over 191 million Africans used social media. In 2017 alone, Kenya saw a 35\% increase in internet users, 1 million new social media users, and a $10 \%$ increase in phone use [10]. One in five Africans (21\%) now regularly get news from social media, and among youth and citizens with post-secondary education, the Internet and social media are more popular sources of news than newspapers [13].

In response, the traditional African media (newspapers, radio, TV, etc.) have expanded their online presence. Kenya's most widely circulated newspaper, The Daily Nation, has over 1.5 million followers on Twitter, and NTV, its sister TV station, has over 2 million followers and posts in both English and Swahili. Several international media groups, including the BBC and Deutsche Welle, have bureaus in Kenya that regularly post content on social media and on their websites in Swahili and English. The spread of the internet and urbanization, however, has fragmented the media landscape [14]. The result is that targeting communications to general audiences has become more difficult. Placing an article on landscape restoration in The Daily Nation no longer guarantees the influence it once did. At the same time, the increasingly fragmented media landscape enables messages to freely reach new and more specific audiences and for communicators to directly shape the narrative.

Access to technology, however, is still a limiting factor to communications, particularly in low-income countries (LICs) and in rural areas, where there is limited access to electricity. Those who live in rural areas, including restoration practitioners, are also less likely to have access to the internet, mobile phones, and other communication technology. Women in Africa are also 13\% less likely to own a mobile phone than men. Another limitation to efficient communication across Africa is its high linguistic diversity [15]. While some researchers estimate that over $50 \%$ of Africans speak more than two languages, fluency in major international languages, especially English and French, is limited, especially in rural areas [16]. For restoration communicators who craft messages in international languages, engaging local audiences is a major challenge. 


\section{The Global Landscapes Forum}

The Global Landscapes Forum (GLF), a space for discussion for policymakers, implementers, and others working to improve landscapes, was originally hosted in 2013 as part of the United National Climate Change Conference of Parties (UNFCCC COP). The GLF, which separated from the UNFCCC in 2017, calls itself "the world's largest knowledge-led platform on sustainable land use," and restoration is one of its key pillars [17]. The GLF serves as a key space where NGOs, governments, the private sector, practitioners, and international organizations can share expertise, network, and pilot new projects or theories of change.

The GLF organized its first Africa-based conference in Nairobi, Kenya at the headquarters of the United Nations Environment Programme (UNEP) from August 29 to 30, 2018. The GLF argued that, "By bringing together actors from all backgrounds and sectors, the conference will spark a global conversation around Africa's landscapes" [1]. The conference, "Landscape Restoration in Africa: Prospects and Opportunities," offered an opportunity to highlight African leadership and focused on boosting diversity and inclusion at the international level, specifically amplifying the voices of African speakers and youth attendees. In this spirit, the GLF sponsored 100 African youth leaders under 35 years old to attend the conference and a separate leadership program [18]. Several of these youth leaders are active and influential on social media, and they used their clout to spread positive messages about sustainable landscapes to their extensive networks during the conference. The Nairobi 2018 GLF drew 800 in-person attendees, and 13,000 people tuned in online. The GLF's social media reached another 52 million people [17].

\section{Social media analysis}

In the past 10 years, text mining of social media data has contributed to research on politics, marketing, public health, urban planning, transportation, and education [19]. Twitter data have been used for a wide variety of applications, including identifying gang members [20], predicting stock market movements [21], and forecasting voter turnout [22]. Within the field of climate change research, Twitter data have primarily been used to monitor public opinion on climate change. For instance, [23] analyzed how exposure to severe weather events affects climate change perceptions on Twitter. They found that users express their concerns related to extreme weather on social media, and that major climate events can drive changes in sentiment and mood on Twitter. In a similar study, [24] concluded that Twitter is an important platform for spreading climate change awareness. These studies point to the utility of mining large-scale social media data to supplement or replace traditional survey methodologies in cases where they are not feasible to conduct because of geographic or temporal scale.

Recent advancements in natural language processing, such as word and sentence embeddings, have significantly increased the utility of text mining approaches to Twitter data. While initial studies primarily relied on latent Bayesian methods like 
Latent Dirichlet allocation [25], the short and highly varied nature of Tweets reduces the accuracy of unsupervised classification with these methods [26]. Embedding methods, such as Word2Vec [27] and GloVe [28], have improved the ability to identify hundreds of topics across large collections of Twitter data [29]. One such method, the Universal Encoder (USE) [30], is particularly relevant to modeling Twitter data because it is designed to be generalizable to a wide range of variable length documents by leveraging multi-task training on multiple downstream tasks like classification, subjectivity and sentiment analysis [30]. Li et al. [31] identified USE as the current state-of-the-art technique for classifying and identifying topics in Twitter data.

Identifying the demographics of users on social media platforms can provide invaluable context to topic identification and network analysis of social media communities. There are a number of ways to infer the demographics of users on Twitter, including computer vision, network analysis, website traffic data, and natural language processing of names and posts. Analyzing first and last names with a character-based recurrent neural network, [32] achieved 73\% accuracy classifying 13 ethnicities, and 83\% accuracy classifying people into four categories (Asian, Hispanic, black, white). Computer vision approaches, such as the wide residual network, have recently enabled researchers to predict the age of people in photographs with an average error of 2.7 years, significantly outperforming human estimation, which averages a 6.3-year error [33]. Deep convolutional neural networks have also achieved $95 \%$ accuracy in image-based gender classification [34].

Demographic analyses of Twitter and other social media data using the methodologies described above have made significant contributions to demographic research in recent years, including applications in researching segregation, political ideologies, tobacco use, international migration, and the spread of foodborne illness [35-37] found that Twitter also provides a platform for mobilization for traditionally underrepresented or marginalized groups. Given the widely accepted racial and class inequality in global environmental movements, globally robust, real-time identification of who is engaging in trending topics on Twitter may provide useful insights into how different populations engage with climate issues [38]. For instance, social interactions on Twitter have been used to infer demographic characteristics like political ideology [39], location [40], income [41], and gender [42].

\section{Methodology}

\section{Social media data sources and collection methods}

Raw data for this study were collected from Twitter and Facebook between 20 August 2018 and 15 September 2018 to identify online activity before, during, and after the GLF conference. For a broad understanding of social media interaction with GLF event materials, we collected engagement metrics on Facebook for 21 web pages promoted in the GLF "Nairobi 2018 social media toolkit" (See supplementary information for full list) [43]. Twitter's public API was used to collect relevant Tweets and user profiles during the study period. 
Our other collection mechanism relied on Twitter's real-time filter stream, which allows users to specify a set of keywords, individuals, and locations to track. Using Twitter's API, we used five separate filter streams to collect Tweets that contained a specified keyword, mentions of key users, were written by a specified individual, or were geolocated to a specified location concurrent with our dictionaries developed by restoration experts (See SI for full list; Table 1). While the sample stream gives a random $(<1 \%)$ sample of content posted to Twitter, the filter stream allowed us to collect all tweets that matched our filters posted to the platform as long as the volume of Tweets did not exceed $1 \%$ of the platform's total volume. To compare the data from the GLF Nairobi, we also analyzed a dataset of 575,930 Tweets collected during the 2017 GLF in Bonn, Germany in December 2017.

\section{Data cleaning}

To reduce the noise inherent to social media analysis, we leveraged social interactions among the individual accounts in our dataset to extract content produced by relevant accounts or accounts that interacted with relevant accounts. Retweets, wherein an individual A rebroadcasts a message authored by individual B to individual A's followers, were used to construct a network of individuals with nodes that represent authors of Tweets, and edges constructing directed links between nodes A and $\mathrm{B}$ that represent node $\mathrm{A}$ having retweeted node $\mathrm{B}$.

Prior work has found that the community structure in retweet networks is predictive of community membership, e.g., for political orientation [39]. We, therefore, used the community structure in our retweet network to form groups of individuals and their discussions. For each sizable community in the overall retweet network, we identified the most retweeted Twitter accounts and the most shared hashtags to discard communities that did not discuss topics relevant to the GLF. As a result, every tweet in our final dataset was filtered both by content (i.e., keywords and accounts) and by social interactions (i.e., the author interacts with a group discussing GLF-related topics). To extract the community structure from this retweet network, we used a label propagation algorithm, which iteratively spreads a node's label to its neighbors, and each neighbor adopts a label based on majority voting.

Table 1 Data collection streams, timeframes, and number of collected Tweets

\begin{tabular}{lllc}
\hline Stream & Description & Timeframe & Tweets \\
\hline 1 & GLF Nairobi 2018 Promoted Hashtags and @ GlobalLF & 21 August-15 September & 19,770 \\
2 & Tweets geolocated to Nairobi, Kenya & 20 August-15 September & 175,819 \\
3 & Accounts from the Global Restoration Council, Global & 21 August-15 September & 710,004 \\
& Partnership on Forest and Landscape Restoration, & & \\
& GLF partners, and GLF-promoted organizations as & & 109,867 \\
& well as African conference voices and youth leaders & & 21 August-15 September \\
5 & High-confidence dictionary of 143 keywords & 21 August-15 September & 90,569 \\
\hline
\end{tabular}


For tractability concerns, prior to running label propagation, we removed nodes with fewer than 2 edges and only stored communities with 100 or more users.

We identified four communities with over 100 users with discussions relevant to the GLF: One community centered on the hashtags "restoration", "climate action", and "climate change", the second focused on the hashtag "\#Plant4Pakistan", the third community was composed of UN agencies, and the fourth community focused on the Mongabay account/climate change. These four communities contained 17,752 unique Twitter accounts and 188,675 tweets.

\section{Identifying important individuals in social network data}

We identified three types of central users among the restoration-related communities: authoritative users, users with the furthest "reach", and users that connect different communities. These types of individuals correspond to three centrality metrics common in social network analysis: PageRank, closeness centrality, and betweenness centrality. An individual's PageRank score is proportional to the likelihood of encountering that individual after an infinite random walk through the network, which we interpret as "authority." Closeness centrality measures the average shortest path to each node, so individuals with high closeness centrality can rapidly distribute information. Lastly, betweenness centrality is a measure of how many shortest paths between all pairs of nodes traverse a given node, corresponding to "bridges" between otherwise disconnected communities. In addition to measuring central users in the social network with centrality measures, we also analyze the "loudest" users, measured by the total relative volume of tweets for each user in the network.

\section{Quantifying influence through effects on followership}

To understand how an organization or an individual can influence the conversation on a social network, we measured the effect that an influential account has on driving other individuals to new content by measuring how the number of Twitter followers of other accounts changes over time. This metric operates under the assumption that significant increases in follower count indicate that an influential account pushed many of its followers to follow a new account. In the context of the GLF Nairobi, this metric also provides insight into whether the event itself leads to greater exposure. That is, if the population taking part in the GLF was already densely connected, one would expect little change in followership for promoted or advertised accounts. If, on the other hand, the GLF engaged new individuals, we would expect increases in followership among promoted and central accounts participating in the GLF discussion. We measure these increases in followership by absolute increase and multiple increase. The former relates to the absolute change in Twitter followers from the beginning to the end of data collection, while the latter is the multiple between the maximum and minimum number of followers for each account during the study period. 


\section{Identifying demographics and locations from twitter data}

A stated goal of the GLF Nairobi was to promote African voices in the restoration movement. To get a sense of the demographics of individuals participating in the GLF discussion on social media, we estimated the age, gender, race, and geographic origin of Twitter profiles using a combination of self-reported location data, computer vision of social media photographs, natural language processing, and inferential statistics of U.S. Census and Wikipedia data.

Self-reported locations on Twitter profiles were mapped to countries using the pigeo Python library [44], which is a machine learning-based classifier trained to infer a user's city and country from a given location string. To compare user locations between the GLF Nairobi and the previous GLF in Bonn, we compared the distribution of user locations in our Twitter datasets for both Nairobi and Bonn. A wide residual network [45] was used to estimate the age and gender of individuals in Twitter photographs. The pre-trained network was originally trained on over 50,000 photographs labeled by age and gender derived from a number of sources, including Wikipedia, social media, and Flickr [33]. The reported validation error for age was 3.96 years and for gender was $12 \%$. We modified the architecture in [33] to automatically identify and disregard Twitter handles that do not have profile pictures containing faces. When there was more than one face, the Twitter handle was assigned the age and gender most likely to belong to the name associated with the handle. Individuals' first names were compared with gender distributions obtained from historical U.S. census data to add robustness to the gender prediction [46]. The name-based and photograph-based gender identification matched in $96 \%$ of sample cases, and non-matching samples were dropped. While we are unable to report a validation F1-score for the combined computer vision and name classification approach due to a lack of labeled response data, it is likely that combining these two metrics and subsetting data to positively matched samples increase the robustness of the classification.

Race was classified into four groups (Asian, African, White, and Hispanic) using a character-level recurrent neural network of first and last names using the architecture put forth by [32]. Asian-classified names included those which were East Asian, Japanese, and Indian as well as Asian registered voters (as identified by the U.S. Census), African names included Greater African and Muslim names and black registered voters, White names included non-Hispanic European names (including British, East European, Jewish, French, Germanic, Italian, and Nordic) and white registered voters, and Hispanic included Hispanic European names as well as Latino registered voters.

A number of computer vision approaches to estimating race were tested, but namebased approaches consistently had higher validation accuracy. The method employed by [32] uses over 12 million names in the Florida voting registration database and over 130,000 unique names from Wikipedia articles. The original paper reports a validation F1-score, measured as the harmonic mean of precision and recall, of 0.83 . Because the original model generalizes US voting data to non-US regions, we improve the robustness of our approach by analyzing results based on a binary white/non-white metric, for which the model reports a $0.90 \mathrm{~F} 1$-score. We also compared results using the methods 
in [32] with the location metrics described above, for which all demographics matched within $2 \%$. Twitter accounts that did not list both their first and last name, as well as those that listed other words than proper nouns, were removed from consideration in the data cleaning stage. After removing Twitter profiles with no photographs or photographs with no identifiable individuals, and Twitter profiles with non-identifiable names, this ensemble approach enabled the automated identification of demographics for $72 \%(12,744)$ of the 17,752 Twitter handles within our dataset, representing 135,512 Tweets.

\section{Identifying topics in Twitter data}

Tweets are typically difficult to analyze with natural language processing, owing to their frequent grammatical and spelling errors, presence of hashtags and emoticons, and short text length. We used the Python module "ekphrasis" to clean Twitter text data, which exploits a character-level recurrent neural network trained on over 1 billion Tweets from 2017 and 2018 [47]. This enabled us to automatically correct spelling errors and elongated words, separate hashtags into constituent phrases, and convert user mentions and other common Tweet structures into a single unique token. To model the semantic and topical structure of Tweets, a number of recent natural language processing approaches were tested. These include latent Bayesian approaches such as latent Dirichlet allocation [25], structured topic models [48], biterm topic models [26], as well as neural embedding approaches including Word2vec [27], Tweet2vec [49], MUSE [50], and the Universal Sentence Encoder [30]. The Universal Sentence Encoder (USE) performed better than the other tested approaches when qualitatively evaluated on sentence similarity and topic coherence.

The Universal Sentence Encoder uses transfer learning to learn task-invariant sentence representations. The pre-trained model uses the transformer architecture [51] to jointly learn tasks including sentiment, subjectivity, and polarity analysis as well as question classification and semantic similarity. This generalizability makes it a strong candidate for representing the topics and meanings of Tweets, which vary widely in diction and prose. 138,512 Tweets from 12,744 unique handles were encoded with the pretrained USE model and clustered with K-nearest neighbors (KNN) clustering. Cluster amounts ranging from 50 to 250 were tested by reading random stratified subsamples of 20 randomly chosen topics. KNN with $k=200$ was selected for final analysis based on this manual validation method. Each of the 200 topics was manually labelled by reading 50 random Tweets in each topic. The age, gender, and racial distribution of each topic were calculated by joining the topic number to the demographic data set, and differences in demographic engagement within topics were calculated with a Chisquare test. Analyses were performed in the R environment for statistical computing [52], as well as Python 3.6 with TensorFlow 1.4 [53]. 
Daily engagement metrics for Twitter and Facebook

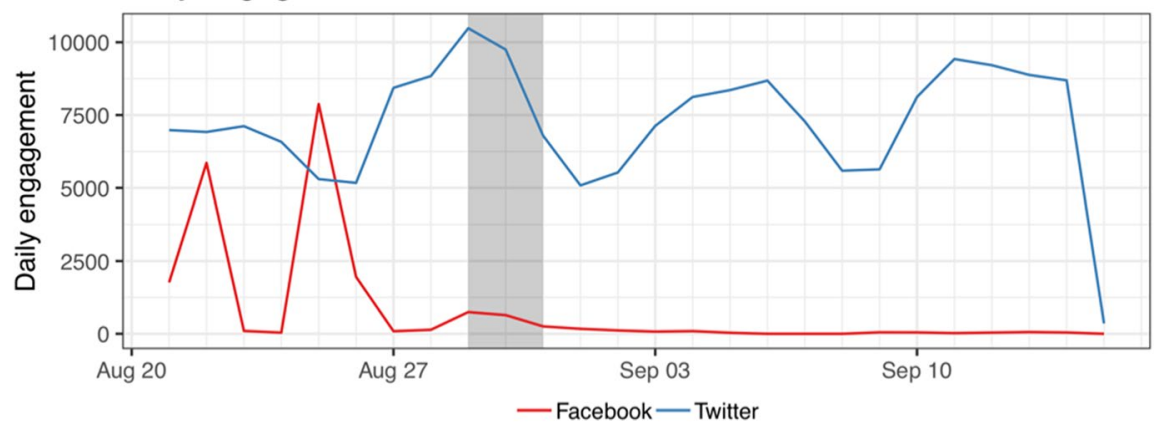

Fig. 1 Reactions to GLF-promoted links on Facebook (red) and Tweets about the GLF (blue) during the study period. The days shaded in gray correspond to the GLF Nairobi event

Table 2 Most frequent, Retweeted, and Central Accounts

\begin{tabular}{llllll}
\hline Rank & Loudest & Retweeted & PageRank & Closeness & Betweenness \\
\hline 1 & OlumideIDOWU & UNEnvironment & UNFCCC & UNFCCC & GlobalLF \\
2 & RichardMunang & UNFCCC & UNEnvironment & UNEnvironment & OlumideIDOWU \\
3 & GlobalLF & BBCAfrica & ErikSolheim & UN & UNEnvironment \\
4 & estherclimate & WorldBank & BBCAfrica & antonioguterres & ClimateWed \\
5 & ClimateWed & ErikSolheim & AfDB_Group & ErikSolheim & estherclimate \\
6 & EWestaway & AfDB_group & PEspinosaC & GlobalLF & RnfrstAlliance \\
7 & msimire & GlobalLF & WorldBank & BBCAfrica & ErikSolheim \\
8 & wandieville & rnfrstalliance & GlobalLF & RnfrstAlliance & RichardMunang \\
9 & Individual account & RichardMunang & antonioguterres & WorldBank & AfDB_Group \\
10 & africa_ci & ClimateWed & JoyceMsuya & UNYouthEnvoy & choi_afdb \\
\hline
\end{tabular}

\section{Results}

\section{Overall engagement}

Engagement with GLF event links on Facebook was highest in the days leading up to the conference, while Tweets about the GLF peaked during the event but maintained longer engagement both during the weeks before and after the event (Fig. 1). Overall, GLF-related links were shared on Facebook 14,314 times, representing about $7.5 \%$ of the overall monitored social media engagement.

\section{Identifying influential Twitter accounts}

Measured with graph theory methods, many of the most influential Twitter accounts were international organizations or their representatives (e.g., UN 
Environment, UNFCCC, Erik Solheim), development banks, and news channels (Table 2). To preserve the privacy of individual Twitter accounts, the names of accounts that are not verified, organizations, or public figures (e.g., journalists, activists, spokespeople, celebrities, and major figures of international organizations) have been masked. Governments and organizations had the highest overall increase in Twitter followers, while individuals and smaller organizations saw large multiplicative increases (Fig. 2). African individuals ranked high in the table with prominent activists, government leaders, and reporters: OlumideIDOWU, a Nigerian climate activist and youth leader, was the loudest user. RichardMunang, a public communicator on environmental issues, estherclimate, a climate action blogger at the UN, msimire, an urban planner and journalist, and wandieville, a journalist who covers agriculture, followed closely behind.

\section{Comparing demographics of the GLF Bonn and the GLF Nairobi}

Figure 3 compares the distributions of continental origins for the Twitter accounts taking part in both the GLF Bonn 2017 and GLF Nairobi 2018. The social media community engaging with the GLF Nairobi contained a significantly higher percentage of individuals from Africa than did the GLF Bonn community. The most common user origins by country at the GLF Nairobi were (in descending order) the United States of America, the United Kingdom, Kenya, Nigeria, and South Africa. In contrast, the GLF Bonn's participants most commonly came from the United States of America, the United Kingdom, India, Kenya, and Canada.

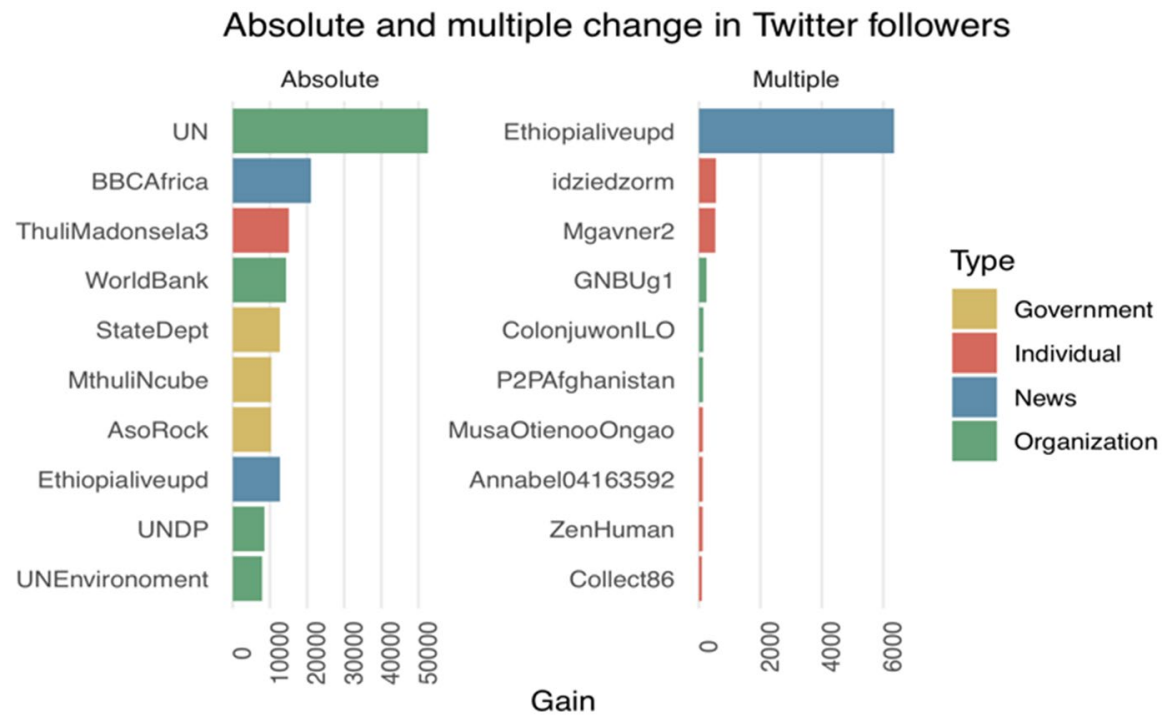

Fig. 2 Absolute (left) and multiple (right) change in Twitter followers during the study period colored by the type of Twitter user 


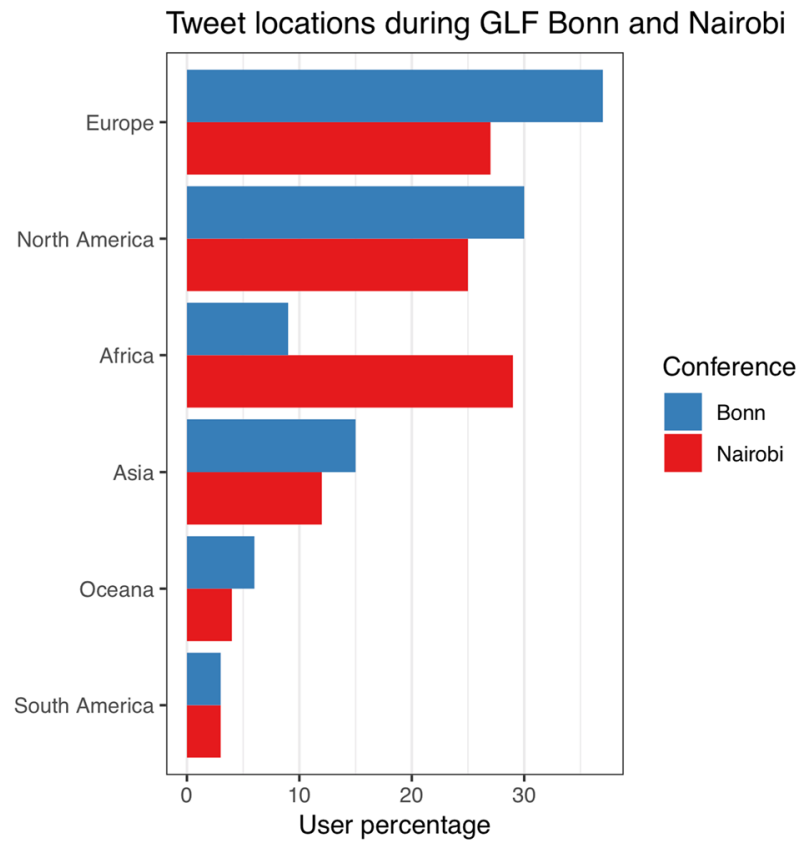

Fig. 3 Continental distribution of studied Twitter users during the Bonn and Nairobi GLF, determined by geolocating user locations from Twitter profiles

\section{Demographic differences in topic engagement}

The most discussed topic on Twitter during GLF Nairobi was retweets of coverage of the latest research, innovation, and publications. Among the top topics were also conversations about the costs of climate change inaction, conferencerelated event information, gender awareness and women in restoration, statements by country governments, and success stories in restoration.

The demographics of profiles interacting with all climate-related topics on Twitter during the GLF Nairobi conference was 59\% men, $41 \%$ women, $25 \%$ African, $16 \%$ Asian, 52\% White, and 7\% Hispanic (Fig. 4). The average age was 30 years, and only $2.5 \%$ were under 24 years of age. There was significant variability in demographic-level engagement with the 200 identified topics. Women engaged significantly more with conversations on natural disasters, endangered species, animal rights, food security, and success stories (Chi-sq $=4573, p<0.01$; Fig. 4). Men engaged significantly more with conversations on supply chains, data and statistics, and illegal activities and corruption. White profiles engaged with conversations on policy, data, research, and the United Nations significantly more than other demographics (Chi-sq $=15,510, p<0.01)$. African profiles engaged significantly more with conversations about youth initiatives, women's rights, industrial pollution, restoration in the supply chain and global market, and the role of technology platforms and apps in tree planting and restoration (Chi$\mathrm{sq}=15,510, p<0.01 ;$ Fig. 4$)$. 

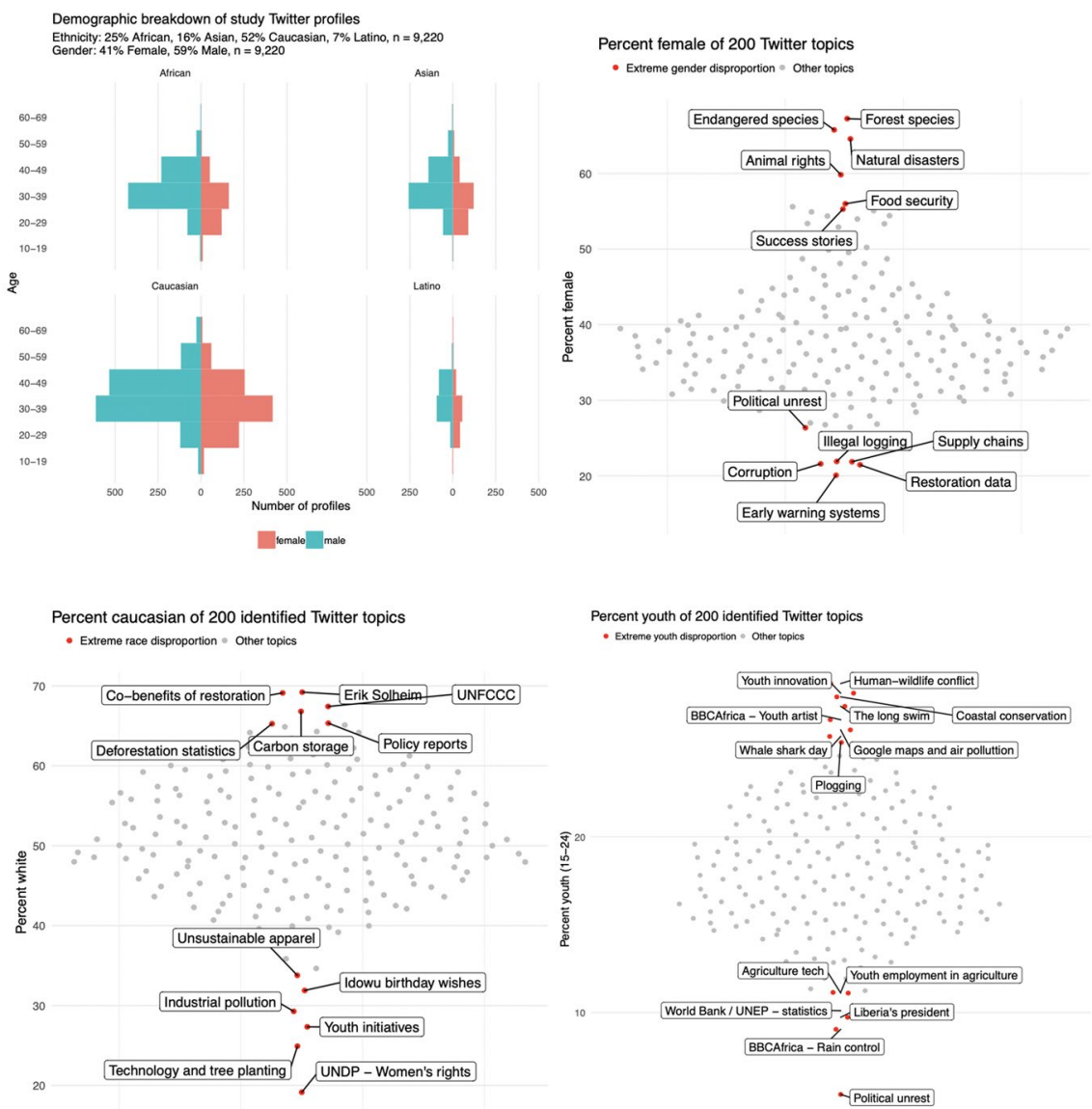

Fig. 4 Top left: demographic breakdown of study Twitter profiles by age, gender, and race. Sample size for gender and age is lower than that of race because the latter does not require a photograph of the user. Top right: topics with disproportionate gender representation. Bottom left: topics with disproportionate race representation. Bottom right: topics with disproportionate youth representation

Youth engagement was significantly higher for viral topics during the event, such as "plogging" - a combination of jogging and picking up litter, news articles about attaching air pollution monitors to Google Street view cars, and "the long swim"- an endurance swim by the UN Patron of the Oceans to raise awareness on ocean pollution (Fig. 4; Table 3). Youth innovation, centering on the youth activities organized by the GLF, had the highest engagement of youth profiles among all identified topics, suggesting that the event increased youth engagement. On the other hand, young people interacted significantly less with conversations on statistics, agricultural technology, and political unrest than older demographics. 
Table 3 Overview of demographic differences in topic engagement on Twitter

\begin{tabular}{|c|c|c|c|}
\hline Demographic & Women & White & Youth \\
\hline More engagement & $\begin{array}{l}\text { + Natural disasters } \\
+ \text { Endangered species } \\
+ \text { Animal rights } \\
+ \text { Food security } \\
+ \text { Success stories }\end{array}$ & $\begin{array}{l}\text { + Policy } \\
+ \text { Data } \\
\text { + Research } \\
\text { + United Nations }\end{array}$ & + Viral topics \\
\hline Less engagement & $\begin{array}{l}\text { - Supply chains } \\
\text { - Data and statistics } \\
\text { - Illegal activities and corruption }\end{array}$ & $\begin{array}{l}\text { - Youth initiatives } \\
\text { - Women's rights } \\
\text { - Industrial pol- } \\
\text { lution } \\
\text { - Restoration in } \\
\text { the supply chain } \\
\text { - Role of technol- } \\
\text { ogy platforms }\end{array}$ & $\begin{array}{l}\text { - Statistics } \\
\text { - Agricultural technology } \\
\text { - Political unrest }\end{array}$ \\
\hline
\end{tabular}

\section{Community network structures between GLF Bonn and GLF Nairobi}

The 2018 Nairobi conference attracted more individuals to the conversation (23,132 vs. 9441 accounts), while the 2017 Bonn conference's smaller network was nearly three times denser (though both networks were quite sparse, at $3.59 \mathrm{e}-4$ and $1.45 \mathrm{e}-4$, respectively). This difference suggests that Nairobi attracted both additional individuals and that these individuals were more likely to engage with less similar and more individuals in the network. Centrality metrics like betweenness and PageRank also suggest that the GLF Nairobi network had more bridges between communities and was less centralized. Recalling that high betweenness centrality suggests the presence of bridges between communities, the 95th percentile for the 2017 GLF in Bonn is about 50\% larger than the 2018 GLF in Nairobi (7.5e-5 to 5.0e-5, respectively). The PageRank, which captures the amount of centralization in the network, of the 2017 GLF is more than double that of the 2018 GLF in Nairobi (1.95e-4 to 8.5e-5, respectively).

In each GLF dataset, we identified four major communities with more than 100 individuals. For the 2017 GLF in Bonn, these four communities centered around restoration organizations, United Nations organizations, proponents of the Rally for Rivers movement, and a conspiracy-theory/anti-climate-change focused group (Fig. 5). For the 2018 GLF in Nairobi, the four communities center on UN organizations combined with restoration groups, Africa-centric organizations, United States politicians/activists, and Pakistani governmental organizations and representatives. The Nairobi GLF network is visibly less centralized and has more bridges between communities, in accordance with the centrality metrics. A full list of the top ten most authoritative individuals in each community is included in the supplementary information. 


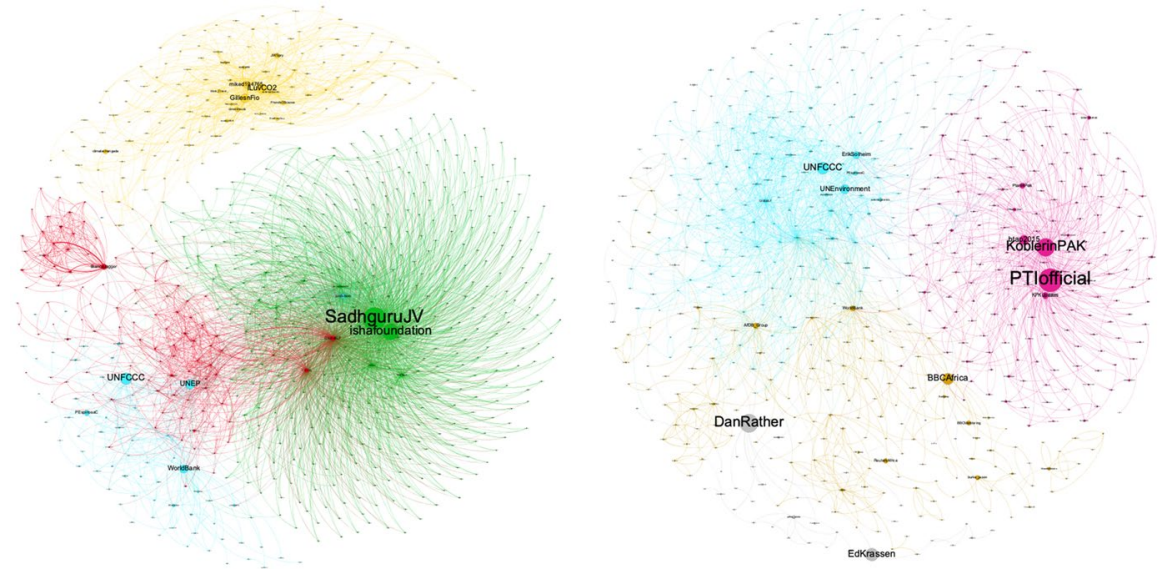

Fig. 5 Community networks of GLF Bonn (left) and Nairobi (right) demonstrating a larger presence of the UNFCC network and a less dense network structure in the Nairobi conference

\section{Discussion}

Analyzing social media data can help researchers to understand how inclusive and influential international events are in real time. The use of machine learning and artificial intelligence for automated classification expands the utility of this data stream by providing automated insights into demographics and discussion topics. For decades, actors in the international environment space have called for increasing voices from developing countries. For organizations like the GLF, which have the explicit goal of engaging and learning from diverse and marginalized groups, analyzing online engagement metrics with machine learning can allow organizers and researchers to quantitatively assess progress toward this goal.

Our results suggest that holding this GLF in Nairobi, Kenya did indeed lead to an increase in engagement of African voices. Afrocentric organizations and African individuals, such as BBC Africa and OlumideIDOWU, identified through location and demographic analysis as well as by the initial Twitter streams, were "louder" than during the 2017 GLF in Bonn, Germany. Kenya, Nigeria, and South Africa featured in the top five countries of origin for the conference. While significant progress was made to include African voices, white males over 30 remained the largest demographic of individuals participating in online discussions.

Of the four communities identified, three focused largely on global or regional agenda, while one community heavily focused on restoration in Pakistan. Although Pakistan is a part of the global restoration movement with a national commitment to the Bonn Challenge, more than $80 \%$ of all forest and landscape restoration in Pakistan is a subnational movement with a specific regional focus [54]. This segmentation of the subnational restoration movement, the "Billion Tree Tsunami" campaign, may explain the presence of this Pakistan-specific community.

Less than three percent of Twitter profiles in our dataset belonged to people under 24 , significantly less than the global Twitter average of $16 \%$. The GLF Nairobi 
hosted a 4-week "Youth in Landscapes Initiative" before and during the conference that was designed to amplify the voices of youth actors in restoration. Our results also suggest that this focus on youth presence at the conference and in online interactions likely increased youth engagement levels on social media, with youth innovation being the top topic that young people interacted with in our dataset. Our results show that youth engaged significantly more with viral topics and significantly less with politics and statistics, indicating that framing communications strategies in ways that appeal to targeted audiences is increasingly important as online conversations continue to fracture. A reactive and adaptive communications strategy that aims to incorporate current trends into messaging could engage more young people.

Users with different demographic characteristics engaged with different topics on Twitter. Our machine learning approach to analyzing social media data allows researchers to monitor how certain messages resonate with specific audiences and can be used to help craft messages that are most likely to resonate with diverse audiences. The strategy of building separate messages aimed at different social groups is successfully used in commercial marketing and advertising [55] and in campaigns that are part of grassroots social movements. In NGO and international organizations, however, it is often not prioritized. Our results demonstrate that the explicit consideration and monitoring of demographic-level engagement metrics may help craft communications strategies that foster increased levels of diversity and inclusion.

\section{Conclusion}

To accelerate restoration throughout Africa, the Global Landscapes Forum brought together a diverse group of practitioners and policymakers at its 2018 event in Nairobi. The GLF succeeded in generating substantial online conversation, reaching over 52 million people on social media surrounding their Nairobi event [56]. Analyzing the conversation on Twitter using machine learning and artificial intelligence for automated classification helps us to understand whose messages and which topics came to the forefront. However, future research is necessary to identify whether the use of online platforms over- or under-estimates offline engagement among different demographic groups based on their access to technology and differing societal norms. While the present paper disambiguates general topics by demographics, the topic of a communications material is often fixed. Future research should consider how to disambiguate demographic engagement within the same topic, for instance by major emotional themes or by verbosity. This would allow communications messaging on a set topic to be tailored to the interests and perspectives of each audience.

Our research shows that choosing to locate the conference in Nairobi did encourage greater participation from youth and local African voices. This finding supports the argument that the GLF must consider location and theme when looking to bring new voices to the conversation. The decision to host a GLF in Accra, Ghana in 2019 is a promising sign. For those looking to encourage greater youth and African participation, our research also highlights concrete steps that communicators can take. Young people, for example, have a strong preference for viral moments or messages 
about technology. A diverse communications strategy is necessary to reach groups that are often sidelined in online conversation and can contribute to a more diverse and inclusive movement.

When considering restoration in Africa, the lack of universal access to electricity and the Internet, especially in the rural areas where those who restore land live, complicates the aspiration for an inclusive African restoration movement. Over $40 \%$ of Africans own a mobile phone, and 725 million will have a smartphone by 2020 [10], but only $37.3 \%$ percent of the population is online [11]. Engaging African audiences online is part of the solution, but we cannot forget the other $60 \%$ of Africans. There is also significant disparity in Internet access between African countries. Kenya has one of the Africa's most developed media and telecommunications landscapes. In poorer countries, like Malawi, with less developed communications tools but where landscape restoration is also at the top of the national agenda, it is significantly more difficult to include local people in the global conversation. Communication strategies must go beyond the digital to ensure that these audiences, often building their own local restoration movements, feel heard and included in decision-making.

\section{Compliance with ethical standards}

Conflict of interest On behalf of all authors, the corresponding author states that there is no conflict of interest.

Open Access This article is licensed under a Creative Commons Attribution 4.0 International License (https://creativecommons.org/licenses/by/4.0/), which permits use, sharing, adaptation, distribution and reproduction in any medium or format, as long as you give appropriate credit to the original author(s) and the source, provide a link to the Creative Commons licence, and indicate if changes were made. The images or other third party material in this article are included in the article's Creative Commons licence, unless indicated otherwise in a credit line to the material. If material is not included in the article's Creative Commons licence and your intended use is not permitted by statutory regulation or exceeds the permitted use, you will need to obtain permission directly from the copyright holder. To view a copy of this licence, visit http://creativecommons.org/licenses/by/4.0/.

\section{Appendix}

See Tables 4, 5, 6 and 7. 
Table 4 GLF Nairobi 2018 URLs Tracked on Facebook

\begin{tabular}{|c|c|}
\hline Page name & URL \\
\hline GLF Homepage & https://www.globallandscapesforum.org \\
\hline GLF Nairobi 2018 Home Page & $\begin{array}{l}\text { https://events.globallandscapesforum.org/nairo } \\
\text { bi-2018/ }\end{array}$ \\
\hline GLF Nairobi 2018 About Page & $\begin{array}{l}\text { https://events.globallandscapesforum.org/nairo } \\
\text { bi-2018/about/ }\end{array}$ \\
\hline GLF Nairobi 2018 Agenda & $\begin{array}{l}\text { https://events.globallandscapesforum.org/nairo } \\
\text { bi-2018/agenda/ }\end{array}$ \\
\hline GLF Nairobi 2018 Theme & $\begin{array}{l}\text { https://events.globallandscapesforum.org/nairo } \\
\text { bi-2018/concept-note/ }\end{array}$ \\
\hline Online Survey for GLF Audience & $\begin{array}{l}\text { https://events.globallandscapesforum.org/nairo } \\
\text { bi-2018/have-your-say/ }\end{array}$ \\
\hline GLF Nairobi 2018 Online Streams & $\begin{array}{l}\text { https://events.globallandscapesforum.org/nairo } \\
\text { bi-2018/join-online/ }\end{array}$ \\
\hline GLF Nairobi 2018 Photo Competition & $\begin{array}{l}\text { https://events.globallandscapesforum.org/nairo } \\
\text { bi-2018/photo-competition/ }\end{array}$ \\
\hline GLF Nairobi 2018 Quiz & $\begin{array}{l}\text { https://events.globallandscapesforum.org/nairo } \\
\text { bi-2018/quiz-landscape-restoration-africa/ }\end{array}$ \\
\hline GLF Nairobi 2018 Resources for Getting Involved & $\begin{array}{l}\text { https://events.globallandscapesforum.org/nairo } \\
\text { bi-2018/shape-the-conversation/ }\end{array}$ \\
\hline GLF Nairobi 2018 Speakers & $\begin{array}{l}\text { https://events.globallandscapesforum.org/nairo } \\
\text { bi-2018/speakers/ }\end{array}$ \\
\hline GLF Nairobi 2018 You Leaders & $\begin{array}{l}\text { https://events.globallandscapesforum.org/nairo } \\
\text { bi-2018/youth-leaders-at-glf-nairobi-2018/ }\end{array}$ \\
\hline GLF Nairobi 2018 News & https://news.globallandscapesforum.org \\
\hline AFR100 Announcement & $\begin{array}{l}\text { http://afr100.org/content/3rd-afr100-annual-partn } \\
\text { ership-meeting }\end{array}$ \\
\hline AFR100 Announcement for the GLF & $\begin{array}{l}\text { http://afr100.org/content/global-landscapes-forum } \\
\text {-nairobi-2018 }\end{array}$ \\
\hline AFR100 Homepage & http://afr100.org/content/home \\
\hline AFR100 Announcement & $\begin{array}{l}\text { http://www.nepad.org/content/3rd-afr100-annua } \\
\text { l-partnership-meeting }\end{array}$ \\
\hline AFR100 Twitter Account & https://twitter.com/Afr100_Official \\
\hline GLF Twitter Account & https://twitter.com/GlobalLF \\
\hline GLF Instagram Account & https://www.instagram.com/globallandscapesforum \\
\hline GLF YouTube Channel & https://www.youtube.com/GlobalLandscapesForum \\
\hline
\end{tabular}


Table 5 Highest gains in followers

\begin{tabular}{lllllr}
\hline Rank & \multicolumn{2}{l}{ Gain in followers, absolute } & & \multicolumn{2}{l}{ Gain in followers, multiple } \\
\cline { 2 - 3 } \cline { 5 - 6 } \cline { 5 - 6 } & Account & Gain & & Account & Gain \\
\hline 1 & UN & 52,521 & & Ethiopialiveupd & 6353 \\
2 & BBCAfrica & 21,052 & & Individual account & 553 \\
3 & ThuliMadonsela3 & 15,094 & & Individual account & 532 \\
4 & WorldBank & 14,352 & & Individual account & 246 \\
5 & Ethiopialiveupd & 12,705 & & Individual account & 146 \\
6 & StateDept & 12,664 & & P2PAfghanistan & 131 \\
7 & MthuliNcube & 10,381 & & Individual account & 127 \\
8 & AsoRock & 10,292 & & MusaOtienoOngao & 122 \\
9 & UNDP & 8511 & & Individual account & 120 \\
10 & UNEnvironment & 7893 & & Individual account & 95 \\
\hline
\end{tabular}

Table 62017 GLF Bonn Communities

\begin{tabular}{lllll}
\hline Rank & $\begin{array}{l}\text { Restoration organiza- } \\
\text { tions }\end{array}$ & UN organizations & $\begin{array}{l}\text { Rally for rivers sup- } \\
\text { porters }\end{array}$ & $\begin{array}{l}\text { Conspiracy/anti-climate } \\
\text { change }\end{array}$ \\
\hline 1 & BiancaJagger & UNFCCC & SadhguruJV & $\begin{array}{l}\text { Suspended account } \\
\text { Individual account }\end{array}$ \\
2 & GlobalLF & UNEnvironment & ishafoundation & Individual account \\
3 & CIFOR & WorldBank & dw_environment & Individual account \\
4 & restoreforward & PEspinosaC & IshaTamil & Individual account \\
5 & FAOForestry & ErikSolheim & jmollins & FriendsOScience \\
6 & mongabay & AfrozShah1 & rallyforrivers & climatefrauds \\
7 & Individual account & KGeorgieva & vishwamTOI & Individual account \\
8 & Individual account & JimYongKim & Individual account & drwaheeduddin \\
9 & FTA_CGIAR & WBG_Climate & Individual account & Individual account \\
10 & Individual account & EmmanuelMacron & IshaUSA & \\
\hline
\end{tabular}

Table 72018 GLF Nairobi Communities

\begin{tabular}{lllll}
\hline Rank & UN Organizations & Afrocentric coverage & US activists & Pakistani organizations \\
\hline 1 & UNFCCC & BBCAfrica & DanRather & PTIofficial \\
2 & UNEnvironment & AfDB_Group & Suspended account & KoblerinPAK \\
3 & ErikSolheim & WorldBank & MichaelAvenatti & btap2015 \\
4 & PEspinosaC & ReutersAfrica & jeffrey_ventre & KPKUpdates \\
5 & GlobalLF & BBCMonitoring & savebutterflies & Plant4Pak \\
6 & antonioguterres & burke_jason & SenBillNelson & Individual account \\
7 & JoyceMsuya & WandileSihlobo & Individual account & Individual account \\
8 & UN & USAID & Individual account & Individual account \\
9 & RichardMunang & ITCnews & MooreCharitable & Individual account \\
10 & RnfrstAlliance & Individual account & Individual account & staoir \\
\hline
\end{tabular}




\section{References}

1. Global Landscapes Forum. (2018) Forest and Landscape Restoration in Africa: Prospects and Opportunities Forest and Landscape Restoration in Africa: Prospects and Opportunities A Global Landscapes Forum event Forest and Landscape Restoration in Africa: Prospects and Opportunities A Globa.

2. Gabizon, S. (2016). Women' s movements' engagement in the SDGs : lessons learned from the Women' s Major Group from the Women' s Major Group. Gender \& Development, 2074(24), 99-110.

3. Carant, J. B. (2017). Unheard voices : A critical discourse analysis of the Millennium Development Goals' evolution into the Sustainable Development Goals Development Goals. Third World $Q ., 38(1), 16-41$.

4. Atzmueller, M., \& Lemmerich, F. (2018). Homophily at academic conferences. In The Web Conference Companion (pp. 3-4).

5. Lovei, M. Desertification is not fate. [Online]. https://blogs.worldbank.org/nasikiliza/desertification -is-not-fate. Accessed 6 Jun 2019.

6. Niang, I. et al. (2014). Africa. In Climate change 2014: Impacts, adaptation, and vulnerability (pp. 1199-1265). Cambridge: Cambridge University Press.

7. TARGET 15-Technical Rationale extended. (2012). [Online]. https://www.cbd.int/sp/targets/ratio nale/target-15/. Accessed 6 Jun 2019.

8. Bonn Challenge. [Online]. http://www.bonnchallenge.org/. Accessed 6 Jun 2019.

9. Yahya, M. Africa's defining challenge I UNDP in Africa. [Online]. http://www.africa.undp.org/ content/rba/en/home/blog/2017/8/7/africa_defining_challenge.html. Accessed 18 Jun 2019.

10. GMSA. The Mobile Economy-Africa 2016. [Online]. https://www.gsma.com/mobileeconomy/ africa/. Accessed 18 Jun 2019.

11. Africa Internet Users. 2019 Population and Facebook Statistics. [Online]. https://www.inter networldstats.com/stats1.htm. Accessed 18 Jun 2019.

12. Mourdoukoutas, E. The hashtag revolution gaining ground. [Online]. https://www.un.org/afric arenewal/magazine/april-2018-july-2018/hashtag-revolution-gaining-ground. Accessed 18 Jun 2019.

13. Nkomo, S., Wafula, A. Strong public support for 'watchdog' role backs African news media under attacklAfrobarometer. [Online]. https://afrobarometer.org/publications/ad85-media_in_ africa_world_press_freedom_day_2016. Accessed 18 Jun 2019.

14. Ramaswamy, A. The big picture: Technology to meet the challenges of media fragmentation. [Online]. https://www.nielsen.com/us/en/insights/reports/2017/the-big-picture-technology-tomeet-the-challenges-of-media-fragmentation.html. Accessed 18 Jun 2019.

15. Heine, B., \& Derek, N. (2000). African languages: An introduction. Cambridge: Cambridge University Press.

16. Wolff, E. (2000). Language and society. In African languages-An introduction (p. 317). Cambridge: CUP.

17. Outcome Statement of the 2016 Global Landscapes Forum: Climate Action for Sustainable Development-Global Landscapes Forum. [Online]. https://www.globallandscapesforum.org/ publication/outcome-statement-2016-global-landscapes-forum-climate-action-sustainable-devel opment/. Accessed 6 Jun 2019.

18. Youth in Landscapes Initiative-Nairobi Leadership Program-Global Landscapes Forum Events. [Online]. https://events.globallandscapesforum.org/nairobi-2018/youth-leaders-at-glfnairobi-2018/. Accessed 6 Jun 2019.

19. Kursuncu, U., Gaur, M., Lokala, U., Thirunarayan, K., Sheth, A., \& Arpinar, I. B. (2019). Predictive analysis on Twitter: Techniques and applications (pp. 67-104)., Lecture notes in social networks Cham: Springer.

20. Balasuriya, L., Wijeratne, S., Doran, D., Sheth, A. (2016). Finding street gang members on Twitter. In 2016 IEEE/ACM International Conferences on Advances in Social Network Analysis and Mining (pp. 685-692).

21. Bollen, J., Mao, H., \& Zeng, X. (2011). Twitter mood predicts the stock market. Journal of Computational Science, 2(1), 1-8.

22. Tumasjan, A., Sprenger, T.O., Sandner, P.G., \& Welpe, I.M. (2010) Predicting elections with Twitter: What 140 characters reveal about political sentiment. In Proceedings of the Fourth International AAAI Conference on Weblogs and Social Media (pp. 178-185). 
23. An, X., Ganguly, A. R., Fang, Y., Scyphers, S. B., Hunter, A. M. \& Dy, J. G. (2014) Tracking climate change opinions from Twitter Data. In $K D D$ (pp. 1-5).

24. Cody, E. M., Reagan, A. J., Mitchell, L., Dodds, P. S., \& Danforth, C. M. (2015). Climate change sentiment on Twitter: An unsolicited public opinion poll. PLoS One, 10(8), 1-18.

25. Blei, D. M., Ng, A. Y., \& Jordan, M. (2003). Latent dirichlet allocation. Journal of Machine Learning Research, 3, 993-1022.

26. Yan, X., Guo, J., Lan, Y. \& Cheng, X. (2013) A biterm topic model for short texts. In Proceedings of the International World Wide Web Conference (pp. 1445-1455).

27. Mikolov, T., Chen, K., Corrado, G.S. \& Dean, J. (2013) Distributed representations of words and phrases and their compositionality. In: Proceedings of the 26th International Conference on Neural Information Processing Systems (pp. 1-9).

28. Pennington, J., Socher, R. \& Manning, C. (2014) GloVe: Global vectors for word representation.

29. Fang, A., Macdonald, C., Ounis, I. \& Habel, P. (2016). Using word embedding to evaluate the coherence of topics from Twitter Data. In Special Interest Group on Information Retrieval.

30. Cer, D. et al. (2018). Universal sentence encoder. arXiv preprint arXiv:1803.11175.

31. Li, H., Caragea, D., Li, X. \& Caragea, C. (2018) Comparison of word embeddings and sentence encodings as generalized representations for crisis Tweet classification tasks. In Proceedings of the ISCRAM Asian Pacific 2018 Conference (pp. 1-13).

32. Sood, G., \& Laohaprapanon, S. (2018). Predicting race and ethnicity from the sequence of characters in a name. arXiv preprint arXiv:1805.02109.

33. Rothe, R., Timofte, R., \& Van Gool, L. (2016) DEX: Deep expectation of apparent age from a single image. In 2015 IEEE International Conference on Computer Vision Workshop. Santiago, Chile.

34. Dhomne, A., Kumar, R., \& Bhan, V. (2018). Gender recognition through face using deep learning. International Conference on Computational Intelligence and Data Science, 132, 2-10.

35. Cesare, N., Grant, C., Hawkins, J. B., Brownstein, J. S. and Nsoesie, E. O. (2017). Demographics in social media data for public health research: Does it matter? In Bloomberg Data for Good Exchange Conference.

36. Cesare, N., Grant, C., Nguyen, Q., Lee, H. \& Nsoesie, E.O. (2017) How well can machine learning predict demographics of social media users?.

37. Murthy, D., Gross, A., \& Pensavalle, A. (2016). Urban Social Media demographics: An exploration of Twitter use in major American cities. Journal of Computer-Mediated Communication, 21(1), $33-49$.

38. Nijbroek, R., \& Wangui, E. (2018). What women and men want: Considering gender for successful, sustainable land management programs. Colombo, Sri Lanka: CGIAR Research Program on Water, Land and Ecosystems (WLE).

39. Conover, M.D., Gonçalves, B., Ratkiewicz, J., Flammini, A. \& Menczer, F. (2011) Predicting the political alignment of Twitter users. In 2011 IEEE Third Int'l Conference on Privacy, Security, Risk and Trust and 2011 IEEE Third Int'l Conference on Social Computing, pp. 192-199.

40. Compton, R., Jurgens, D., \& Allen D. (2014). Geotagging one hundred million Twitter accounts with total variation minimization. In 2014 IEEE International Conference on Big Data, Washington DC, USA.

41. Preoţiuc-Pietro, D., Volkova, S., Lampos, V., Bachrach, Y., \& Aletras, N. (2015). Studying user income through language, behaviour and affect in social media. PLoS One, 10(9), e0138717.

42. Volkova, S. \& Yarowsky, D. (2014). Improving gender prediction of social media users via weighted annotator rationales. In NIPS 2014 Workshop on Personalization, Montreal, Canada.

43. GLF. GLF Nairobi: Social Media Toolkit [Online]. https://events.globallandscapesforum.org/nairo bi-2018/social-media-toolkit/. Accessed 6 June 2019.

44. Rahimi, A., Cohn, T., \& Baldwin, T. (2016). Pigeo: A python geotagging tool. In Proceedings of the 54th Annual Meeting of the Association for Computational Linguistics. (pp. 127-132), Berlin, Germany.

45. Zagoruyko, S. and Komodakis, N. (2016). Wide residual networks.

46. Mullen, L., Blevins, C. \& Schmidt, B. (2018) gender: Predict Gender from Names Using Historical Data. R package version 0.5.2.

47. Joulin, A., Grave, E., Bojanowski, P., \& Mikolov, T. (2016). Bag of tricks for efficient text classification. In Proceedings of the 15th Conference of the European Chapter of the Association for Computational Linguistics, 2(1), 427-431. Valencia, Spain. 
48. Roberts, M.E., Stewart, B.M., Tingley, D., \& Airoldi, E.M. (2013). The structural topic model and applied social science. In NIPS 2013 Workshop on Topic Models: Computation, Application, and Evaluation. Lake Tahoe, USA.

49. Vosoughi, S., Vijayaraghavan, P., \& Roy, D. (2016). Tweet2Vec: Learning tweet embeddings using character-level CNN-LSTM encoder-decoder. In Special Interest Group on Information Retrieval. Pisa, Italy.

50. Conneau, A., Lample, G., Ranzato, M.A., Denoyer, L. \& Jégou, H. (2018) Word translation without parallel data. In: International Conference on Learning Representations (pp. 1-14).

51. Vaswani, A. et al. (2017). Attention is all you need. In 31st Conference on Neural Information Processing Systems. Long Beach, CA, USA.

52. Core Team, R. (2013). R: A language and environment for statistical computing. Vienna: R Core Team.

53. Abadi, M. et al. (2016) TensorFlow: A system for large-scale machine learning. In 12th USENIX Symposium on Operating Systems Design and Implementation.

54. IUCN. Pakistan's Billion Tree Tsunami restores 350,000 hectares of forests and degraded land to surpass Bonn Challenge commitment, 2017. [Online]. https://www.iucn.org/news/forests/201708/ pakistan's-billion-tree-tsunami-restores-350000-hectares-forests-and-degraded-land-surpass-bonnchallenge-commitment.

55. Jansen, B. J., Moore, K., \& Carman, S. (2013). Evaluating the performance of demographic targeting using gender in sponsored search. Information Processing \& Management, 49, 286-302.

56. Global Landscapes Forum Nairobi 2018: The highlights. [Online]. https://events.globallandscape sforum.org/nairobi-2018/. Accessed: 18 Jun 2019.

Publisher's Note Springer Nature remains neutral with regard to jurisdictional claims in published maps and institutional affiliations. 